\title{
A Coupled Map Lattice model for geomagnetic polarity reversals that exhibits realistic scaling
}

\author{
Masayuki Seki ${ }^{1}$ and Keisuke Ito $^{2 *}$ \\ ${ }^{1}$ Department of Liberal Arts, Kobe Tokiwa Junior College, 2-6-2 Otani-cho, Nagata, Kobe 653-0838, Japan \\ ${ }^{2}$ The Graduate School of Science and Technology, Faculty of Science, Kobe University, Rokkoudai, Nada, Kobe 657-8501, Japan
}

(Received August 10, 1998; Revised April 28, 1999; Accepted July 7, 1999)

\begin{abstract}
Seki and Ito (1993) showed that the geomagnetic polarity reversals had a power-law distribution and presented a simple model in which the geodynamo was assumed to be a system of magnetic spins in a critical phase-transition state. We present an improved, more realistic model, and obtain a power exponent in agreement with the observed value, which is about -1.5. The revised model is a Coupled Map Lattice (CML). A CML is a dynamical system with discrete time and space, but continuous state. In the present model, elementary dynamo evolves autonomously according to the Lorenz map obtained from Rikitake dynamo dynamics. We examine the behavior of the system and the distribution of polarity reversal intervals for various values of parameters. We find some sets of the parameters which yield a power exponent close to -1.5 .
\end{abstract}

\section{Introduction}

The geomagnetic field has reversed repeatedly in earth's history. For understanding this phenomenon, researchers improve methods of data acquisition, analyze these data and examine the statistical properties. Kono (1987) stated that exponential distribution of polarity reversal lengths is one of the most important statistical properties of the geomagnetic field. This property was originally suggested by Cox (1968). Naidu (1971) showed that gamma distribution provides better fit to the observed intervals of the Cenozoic time scale than exponential one. McFadden (1984) suggested that the underlying process of geomagnetic reversals may be Poissonian rather than gamma process because incomplete data from a non-stationary Poisson process leads to a distribution indistinguishable from a gamma distribution.

Reseachers of geomagnetism traditionally examine the nature of paleomagnetic polarity data divided into two groups separated by the Cretaceous quiet epoch. Gaffin (1989) analyzed the geomagnetic polarity reversal record for the past 165 m.y., and found that the cumulative distribution of the polarity intervals follows a power law. He concluded, however, that the apparent scaling does not warrant selfsimilarity and demonstrated that the non-stationary Poisson model (McFadden and Merrill, 1984; Lutz and Watson, 1988) provides an adequate description for the distribution of interval lengths. Seki and Ito (1993) obtained a similar result to Gaffin (1989), but interpreted the power law distribution as an evidence of the dynamical phase-transition state of geodynamo.

* Now at The Hakodate Wide Area Unification of Public University 4-13 Shinonome-cho, Hakodate, 040-0036, Japan.

Copy right (C) The Society of Geomagnetism and Earth, Planetary and Space Sciences (SGEPSS); The Seismological Society of Japan; The Volcanological Society of Japan; The Geodetic Society of Japan; The Japanese Society for Planetary Sciences.
The important questions are if the geodynamo process is stationary, or if the outliers in the distribution of polarity intervals are to be included in a single model or to be attached to some fundamentally different state. We take the view that a model is good if the total distribution can be explained by the single model without assuming the existence of different states.

Seki and Ito (1993) stressed some peculiar properties of a stable distribution with a form of power-law like

$$
\mathrm{P}(x) \propto x^{-\alpha-1} \quad(0<\alpha<2)
$$

with $\alpha=1.5$ in the polarity interval distribution. In particular, the mean value as well as the variance of $x$ fluctuate strongly as time advances (Takayasu, 1990). Such a process is statistically non-stationary but is dynamically stationary, occurring in a single physical state.

A typical model which exhibits the power-law distribution is self-organized criticality (SOC) model proposed by Bak et al. (1987). Appilcatons of the idea of SOC to various kinds of natural phenomena are reviewed in Bak (1996). The model with proper injection and dissipation of energy tends to self-organize into a critical state independent on the initial condition, Sornette (1992) extended the mechanism of SOC and proposed a general model of open system with a feedback of the order parameter onto its control parameter.

The model in Seki and Ito (1993) has a similar to Sornette's model feedback mechanism and exhibits critical behavior like the power-law distribution of the polarity reversal intervals. The behavior of model, however, differed from that of geomagnetic data in the power exponent being -0.5 , while the power exponent obtained from geomagnetic data was -1.5 . In the present paper, we present an improved, more realistic model, in which its power exponent is in agreement with the observed value. 


\section{Explanation of CML Model}

In our previous paper, we used data from Harland et al. (1982). After their work, various researchers estimated new time scales. Thus, we retried to plot a cumulative distribution of polarity reversal length on a log-log graph (Fig. 1). Here, the time scale from late Cretaceous to present is like in Cande and Kent (1995), and before 118.0 Ma, we have modified the scale of Channell et al. (1995) to be consistent with the one by Cande and Kent (1995). We can regard the distribution in Fig. 1 as a power-law using the latest data about the time scales. The exponent of power in new geomagnetic data is about -1.5 .

Since the distribution of geomagnetic reversal length appears to follow a power-law, we take a power law as a working model. We presented a simple model in which turbulent eddies in the outer core were assumed to behave as magnetic spins (Seki and Ito, 1993), and obtained a power-law distribution for the interval of polarity reversals with the power exponent being -0.5 . The exponent from geomagnetic data, however, was -1.5 . We thought that this discrepancy was due to the fact that our model was oversimplified with respect to the actual geodynamo. We tried to improve the model in order to make it more realistic and to obtain the power exponent to be closer to the observed value. The previous model had discrete time, space, and states. At first, we examined behavior of spin systems on fractal lattices of various fractal dimension, since if the convection of the outer core of the earth is turbulent (de Wijs et al., 1998), then the distribution of turbulent eddies is self-similar. However, the change from regular to fractal lattice did not have significant effect on the power exponent of polarity intervals distribution.

In the present paper, we examine another model which consists of dynamical elements with continuous state. Such a model is generally called "Coupled Map Lattice" (See Kaneko, 1993). The model in Seki and Ito (1993) had twostates ( 1 or -1$)$ elements like Ising model, whereas elements of the present model have continuous state values varying be-

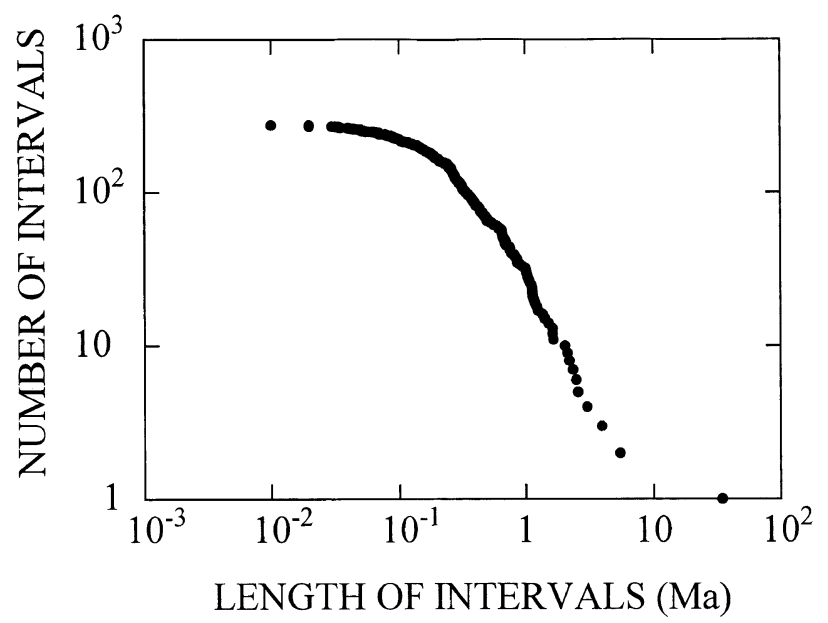

Fig. 1. Log-log plot of the cumulative distribution of polarity reversal length using data from Cande and Kent (1995) and Channell et al. (1995). tween 1 and -1 . Moreover, the elements themselves evolve and reverse the polarity autonomously according to Rikitake dynamo dynamics (Rikitake, 1958).

The differential equations of Rikitake dynamo are ex-

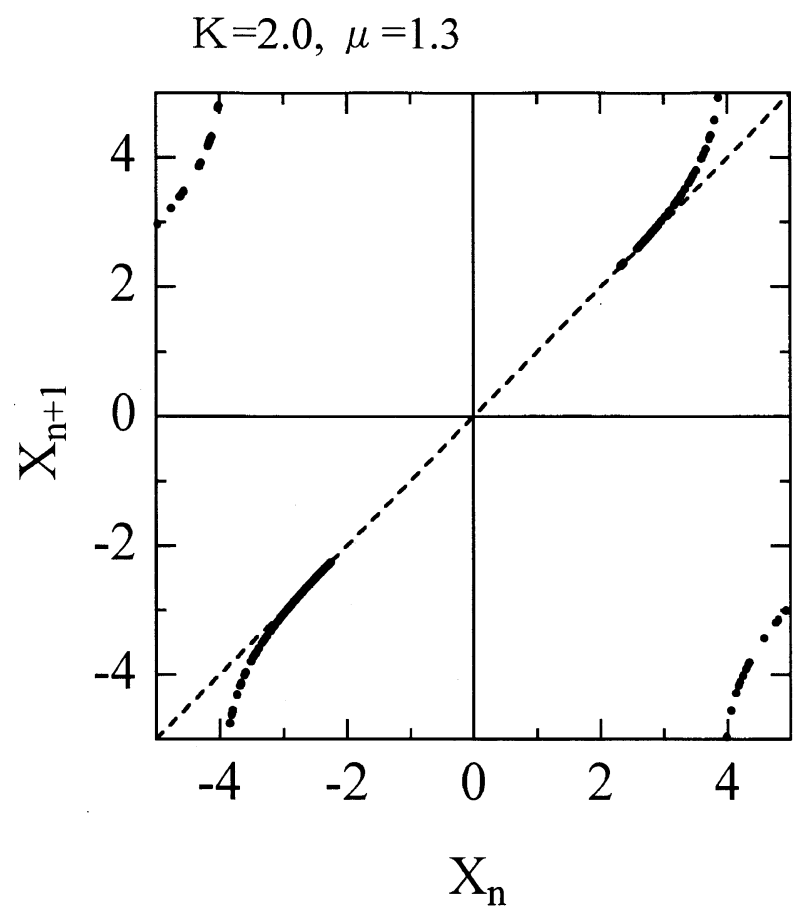

Fig. 2. The Lorenz map of Rikitake Dynamo system $(K=2.0, \mu=1.3)$.

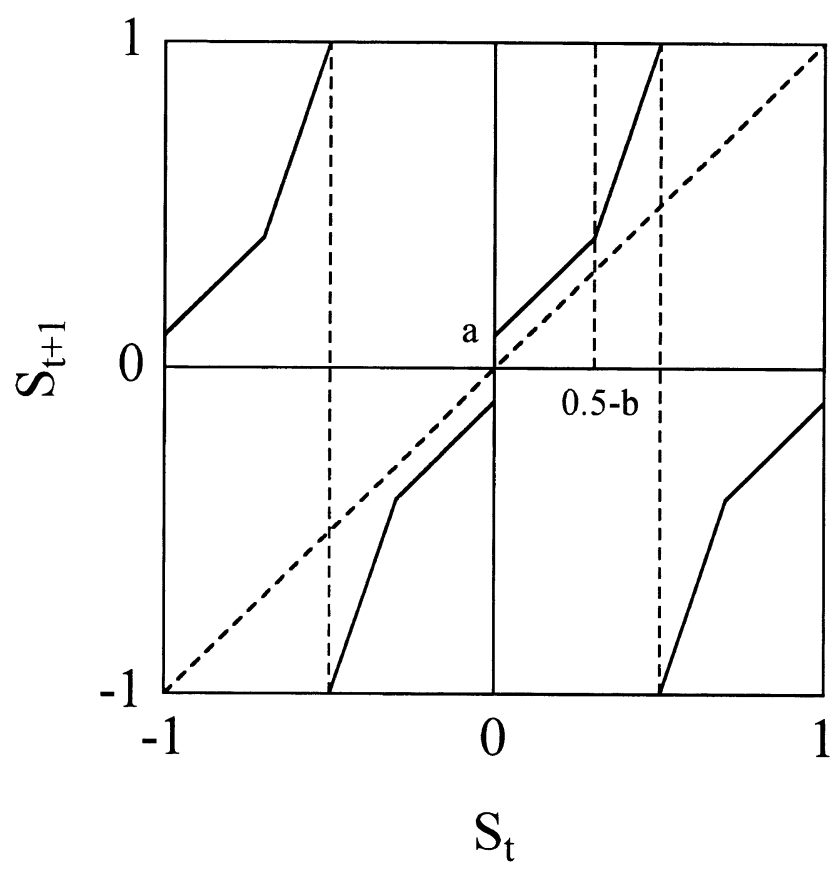

Fig. 3. The piece-wise linear Lorenz map of Rikitake dynamo which we use in CML model. 
(a) $\mathrm{a}=0.01, \mathrm{~b}=0.45$
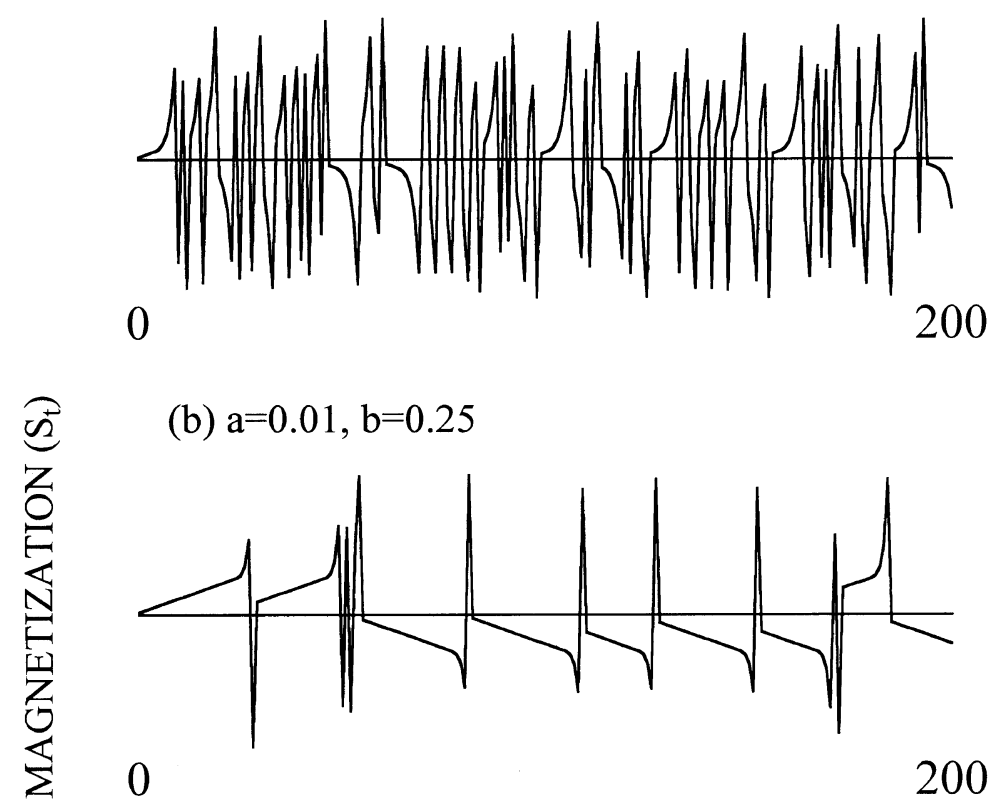

(c) $\mathrm{a}=0.001, \mathrm{~b}=0.45$

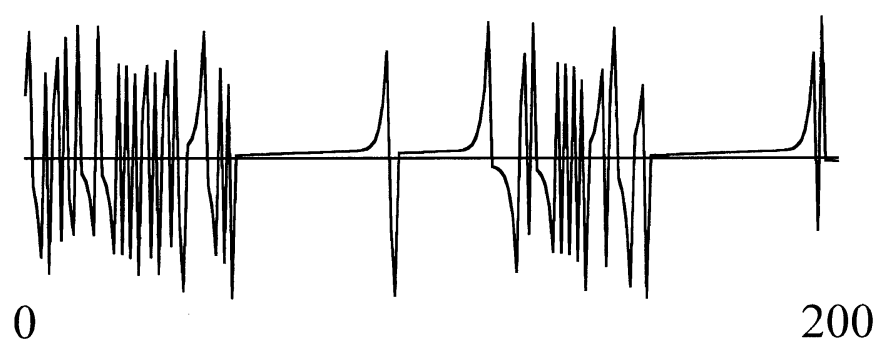

TIME STEP

Fig. 4. The variation of magnetization in elements represented by piece-wise linear Lorenz map of Rikitake dynamo for 200 steps, each case being (a) $a=0.01, b=0.45$, (b) $a=0.01, b=0.25$ and (c) $a=0.001, b=0.45$.

pressed in a non-dimensional form as

$$
\begin{aligned}
\dot{x_{1}}+\mu x_{1} & =x_{2} y_{1} \\
\dot{x_{2}}+\mu x_{2} & =x_{1} y_{2} \\
\dot{y_{1}}=\dot{y_{2}} & =1-x_{1} x_{2} .
\end{aligned}
$$

From (3), we get:

$$
y_{2}=y_{1}+\alpha
$$

where, $x_{1}$ and $x_{2}$ are the currents of dynamo coils, $y_{1}$ and $y_{2}$ are the angular velocities of the dynamo discs. The Rikitake system has two equilibrium points $N$ and $R$ $\left( \pm K, \pm K^{-1}, \mu K^{2}\right)$ in the $x_{1}-x_{2}-y_{1}$ phase space, where $K$ is given by

$$
-\alpha=\mu\left(K^{2}-K^{-2}\right) .
$$

Shimizu and Honkura (1985) proposed $N$-coupled disk dynamo models, but did not try to find the condition of phase transition at which the distribution of polarity reversal intervals is expected to be of power-law type. The computing time that is necessary to solve large sets of differential equations is generally too long, especially near bifurcation points. To reduce computing time, we adopt a one-dimensional map with chaotic behavior based on the differential equations of Rikitake dynamo. Ito (1980) carried out a detailed study of its behavior in the $(\mu, K)$ space, and showed that for a wide combination of $\mu$ and $K$, Rikitake dynamo system exhibits chaos of the Lorenz (1963) type, characterized by irregular flipping between two unstable fixed points (Fig. 2).

Figure 2 shows a Lorenz map of Rikitake dynamo system with chaotic behavior. Lorenz map is a graph which plots combinations of $n$-th local maximum of $|x|$ and $n+1$-th one. We simplify the graph in Fig. 2 into a piece-wise linear form connecting linear functions in $[-1,1]$ (Fig. 3 ). The map is 


\section{EFFECT OF PARAMETER $p$}

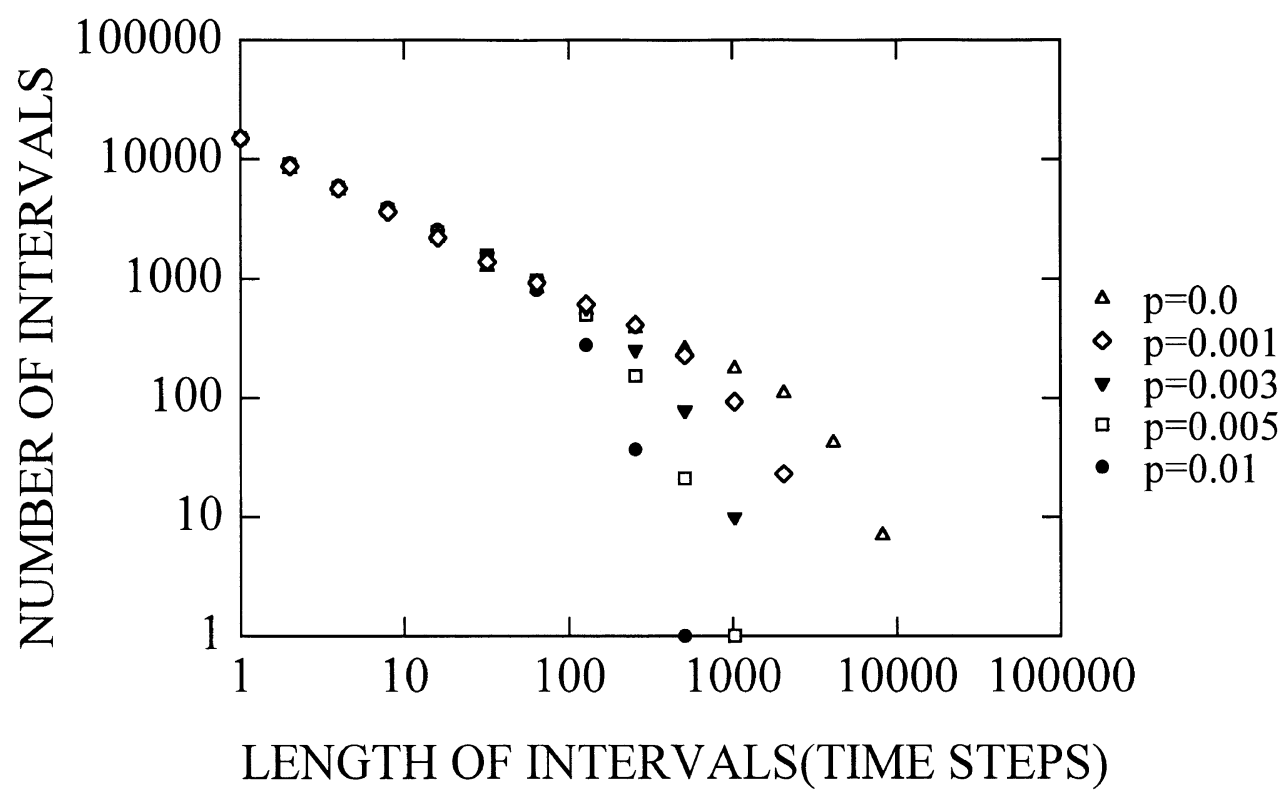

Fig. 5. The effect of parameter $p$ on the distribution of polarity reversal intervals in systems $(16 \times 16)$. Other parameters are fixed; $a=0.0001, b o=0.25$, and $b=0.25$.

given by

$$
S_{t+1}=\left\{\begin{array}{lr}
S_{t}-1-a & \left(\frac{1}{2}+b, 1\right], \\
\frac{1+2 b-2 a}{2 b}\left(S_{t}-\frac{1}{2}\right)-1 & \left(\frac{1}{2}, \frac{1}{2}+b\right], \\
\frac{1+2 b-2 a}{2 b}\left(S_{t}-\frac{1}{2}\right)+1 & {\left[\frac{1}{2}-b, \frac{1}{2}\right],} \\
S_{t}+a & {\left[0, \frac{1}{2}-b\right),} \\
S_{t}-a & {\left[b-\frac{1}{2}, 0\right),} \\
\frac{1+2 b-2 a}{2 b}\left(S_{t}+\frac{1}{2}\right)-1 & {\left[-\frac{1}{2}, b-\frac{1}{2}\right),} \\
\frac{1+2 b-2 a}{2 b}\left(S_{t}+\frac{1}{2}\right)+1 & {\left[-\frac{1}{2}-b,-\frac{1}{2}\right),} \\
S_{t}+1+a & {\left[-1,-\frac{1}{2}-b\right) .}
\end{array}\right.
$$

Parameter $a$ controls the condition of laminar phase of motion (Bergé et al., 1984), and parameter $b$ controls the width of a domain where chaotic bursts occur. Parameter $b$ determines the intensity of chaos. If $b=0$, then the Lyapunov exponent is 0 , and if $b=0.5$ and $a=0$, then the Lyapunov exponent is $\log _{\mathrm{e}} 2$. Parameter $a$ determines the length of laminar phase of intermittency. The closer is $a$ to 0 , the longer is the interval of non-chaotic state. Similar to Ising model, we consider a two-dimensional regular lattice with nearest-neighbor interaction. The state of the site $(i$, $j)$ is represented by $S_{i, j}\left(-1 \leq S_{i, j} \leq 1\right)$. The interaction between elements depends on the summation over the states of four nearest neighbors, sum,

$$
\text { sum }=\sum S_{i \pm 1, j \pm 1} .
$$

We denote the intensity of interaction by $b o$, and take the following procedure of spin flipping. If $S_{i, j} \times \operatorname{sum}<0$, and $\left|S_{i, j}\right|<\mid$ bo $\times$ sum $\mid$, then $S_{i, j}=-S_{i, j}$. If $\left|S_{i, j}\right| \geq \mid$ bo $\times$ sum $\mid$, then $S_{i, j}=-S_{i, j}$ with probability $p_{d}$ which represents a kind of thermal fluctuation. If $S_{i, j} \times \operatorname{sum} \geq 0$, then $S_{i, j}=-S_{i, j}$ with probability $p_{i}$.

$p_{i} \leq p_{d}$, which also represents a simplified thermal fluctuation. In the following simulation, we assume $p_{i}=p_{d}$.

In the present model, the elements reverse the polarity autonomously as well as through interaction with the neighboring elements. Note, that the elements in the previous model changed the sign only due to interaction. It is therefore expected that the present system may exhibit a behavior similar to real data, when short-term polarity reversals occur more often than in the previous model.

\section{Results of CML Model}

The simulation was done using mainly $16 \times 16$ and $32 \times$ 32 square lattice systems with periodic boundary condition. The initial state was given by random numbers. The regular lattice was divided to two sublattices as in the previous model. One step represents a pair of each one procedure of spin flopping on the two sublattices. We examined the behavior for $1.0 \times 10^{6}$ steps, and took the data of polarity reversal intervals within 14999 samples discarding the first $2.0 \times 10^{5}$ steps. Behaviors differ, depending on parameters of the map as well as the probability.

Parameter $b$ controls the width of a chaotic bursts. The larger is $b$, the more frequently polarity reverses, and shorter are the intervals (Figs. 4(a) and 4(b)). Parameter $a$ controls 
EFFECT OF PARAMETER bo

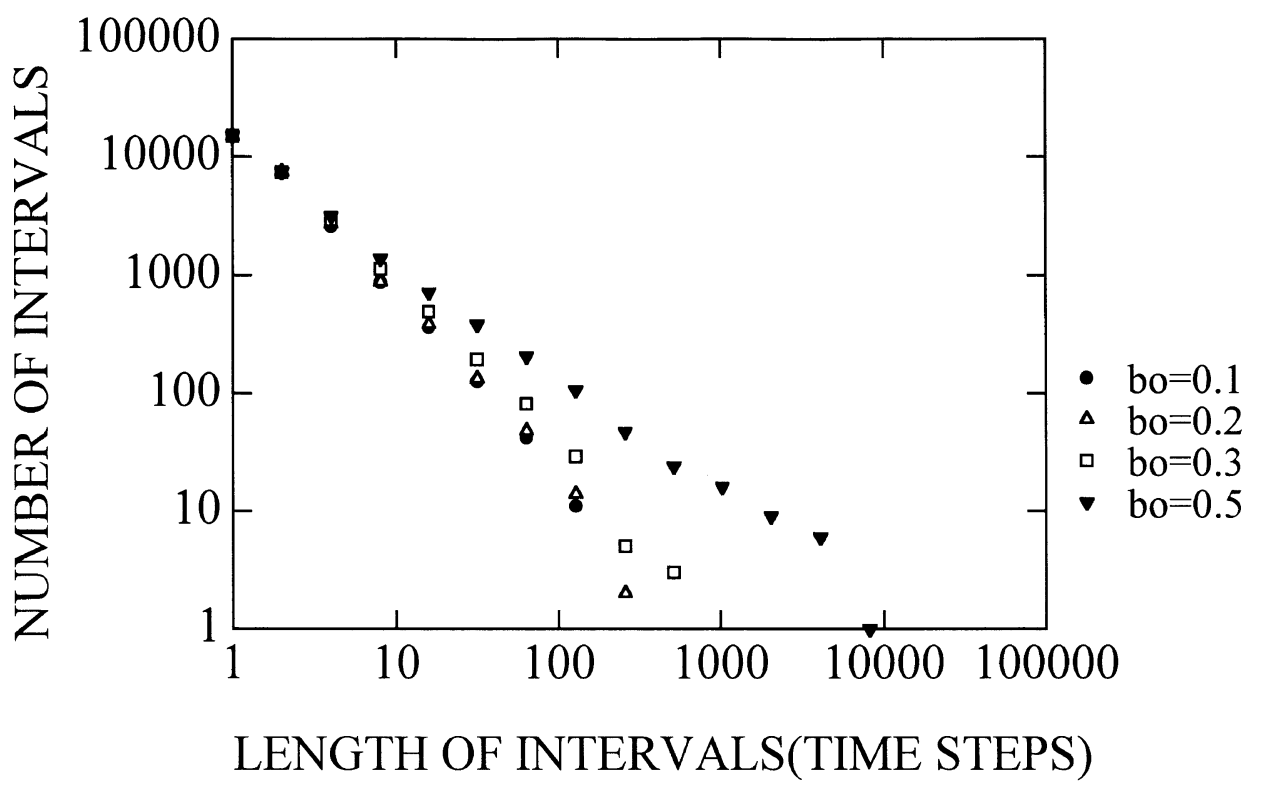

Fig. 6. The effect of parameter $b o$ on the distribution of polarity reversal intervals in systems $(16 \times 16)$. Other parameters are fixed; $a=0.00001, p=0.0$, and $b=0.45$.

(a) $\mathrm{a}=0.0001, \mathrm{bo}=0.1, \mathrm{p}=0.0, \mathrm{~b}=0.2$

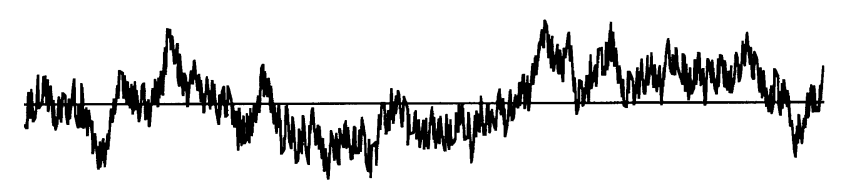

0

25000

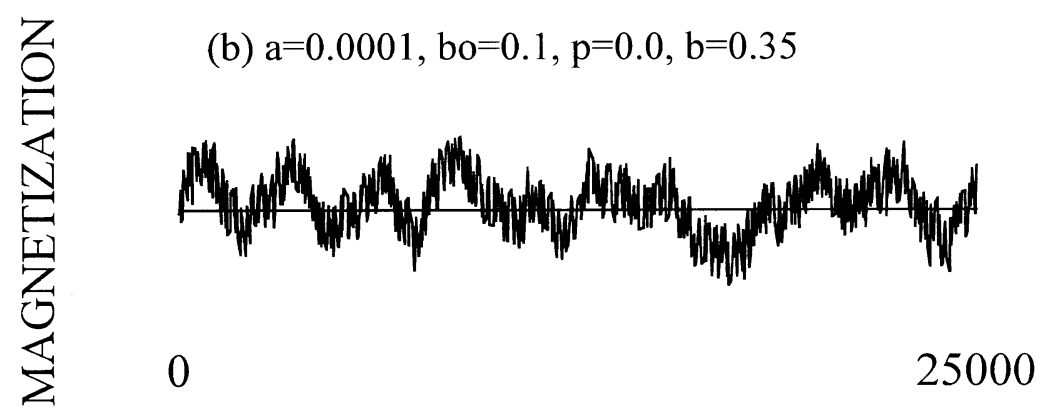

(c) $\mathrm{a}=0.0001, \mathrm{bo}=0.1, \mathrm{p}=0.0, \mathrm{~b}=0.4$

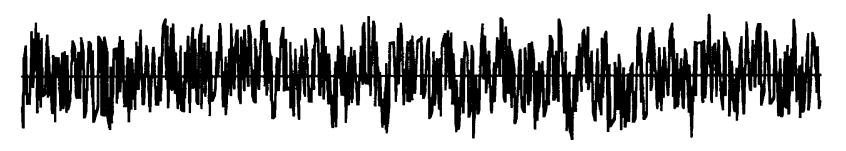

0

25000

\section{TIME STEP}

Fig. 7. The variation of magnetization in systems $(16 \times 16)$ for 25000 time steps. Each case has different value of $b$; (a) $b=0.2$, (b) $b=0.35$ and (c) $b=0.4$. 


\section{EFFECT OF PARAMETER b}

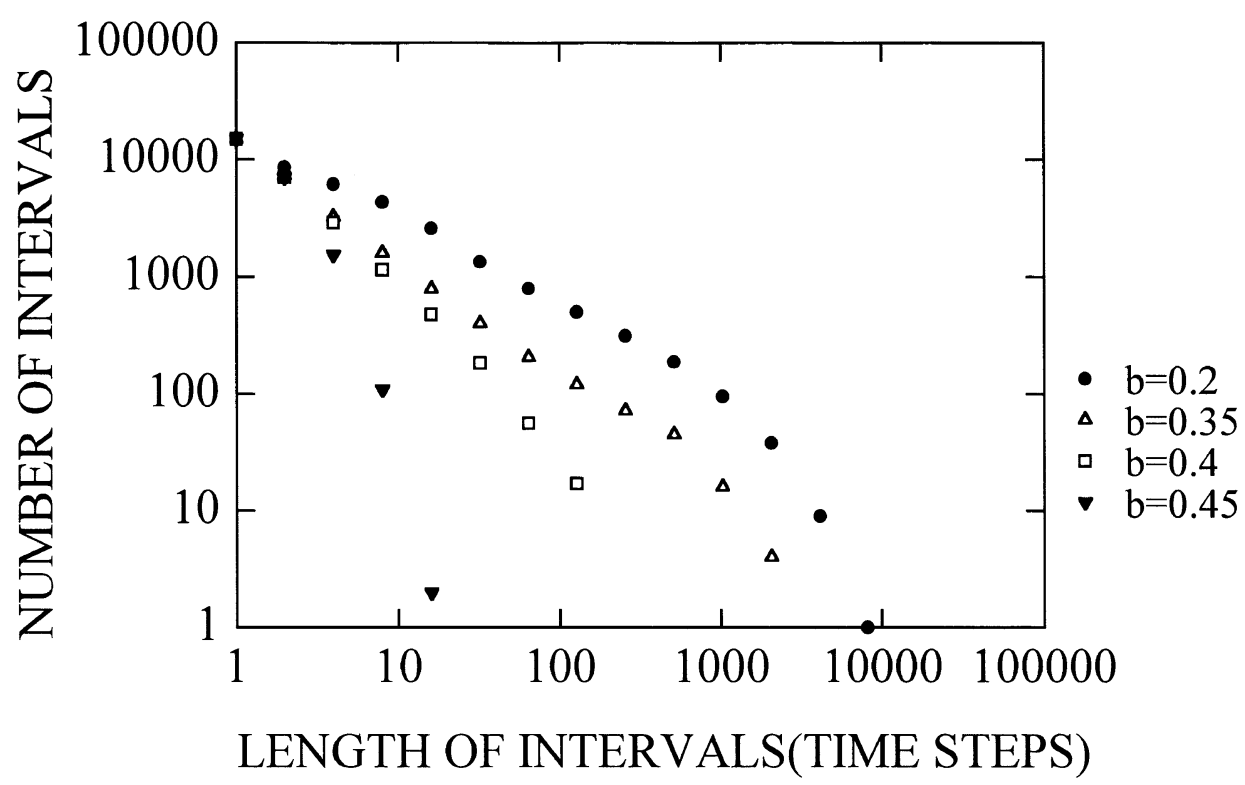

Fig. 8. The effect of parameter $b$ on the distribution of polarity reversal intervals in systems $(16 \times 16)$. Other parameters are fixed; $a=0.0001, b o=0.1$, and $p=0.0$.

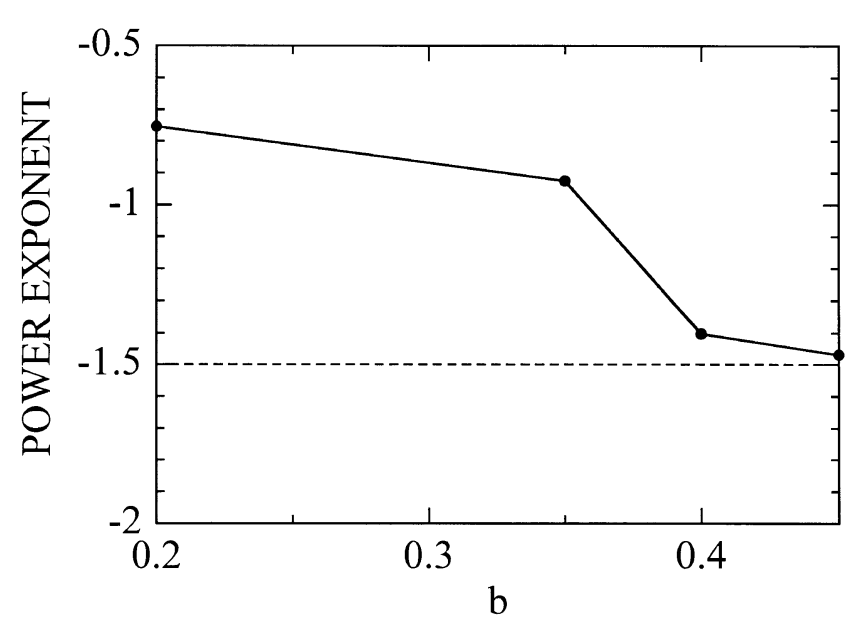

Fig. 9. The power exponent of the distribution of polarity reversal intervals in systems $(16 \times 16)$ as a function of parameter $b$. Here other parameters $a, p$ and $b o$ are fixed at $a=0.0001, p=0.0$ and $b o=0.1$ for $b=0.2$, 0.35 , and 0.4 . For $b=0.45, a=0.00001, p=0.0$ and $b o=0.1$.

the laminar domain. In this domain the absolute value of $S$ shows a monotonous gradual increase. The behavior of elemental map (Fig. 3) is very sensitive to the change of $a$. The smaller is $a$, the longer is polarity reversal interval (Figs. 4(a) and 4(c)). It should be kept in mind that $S_{t}$ is not a graph of continuous change in the strength of magnetization but represents the change in the amplitude of oscillations. When we change $a$, we observe a similar phase transition from periodic to chaotic behavior in the distribution of polarity reversals in elementary systems as in the whole system.

Next we examine the effect of control and probability parameters on the behavior of magnetization and distribution

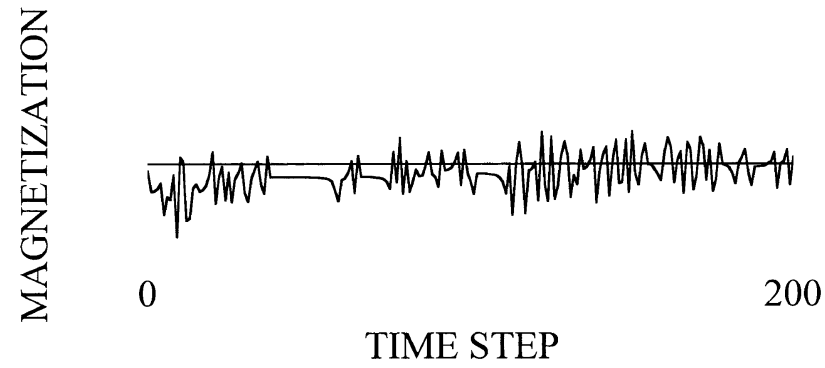

Fig. 10. The variation of magnetization of a system $(16 \times 16)$ for 200 steps. It has the exponent near -1.5 . Here $a=0.00001, b o=0.1, p=0.0$ and $b=0.45$.

of polarity reversal intervals in CML model. As for $p_{i}$ and $p_{d}$, we assume $p_{i}=p_{d}=p$ for simplicity. It is considered that $p$ corresponds to a kind of temperature. When $p$ is small (low-temperature), long intervals of polarity reversals increase in accordance with the supercritical $\left(<T_{c}\right)$ behavior of the previously considered model. When $p$ is large (high-temperature), long intervals of polarity reversal vanish in accordance with the subcritical $\left(>T_{c}\right.$ ) behavior (Fig. 5). Changes in parameter $p$ have little effect on the power exponent. Parameter bo expresses the intensity of interaction between turbulent dynamos. The larger is $b o$, the more ordered the system is. As a result, systems with large bo behave solid-like, showing a supercritical character. The slope of distribution plot tends to be flatter (Fig. 6). As to the effect of $b$ on element's dynamics (Fig. 7), we find that magnetization of the system shows strongly chaotic behavior like white noise and intervals of polarity reversals become short for large $b$ (Fig. 7(c)). Such a tendency is clear in the distribution of polarity reversal intervals (Fig. 8). The larger 
(a) $b=0.4, b o=0.1$

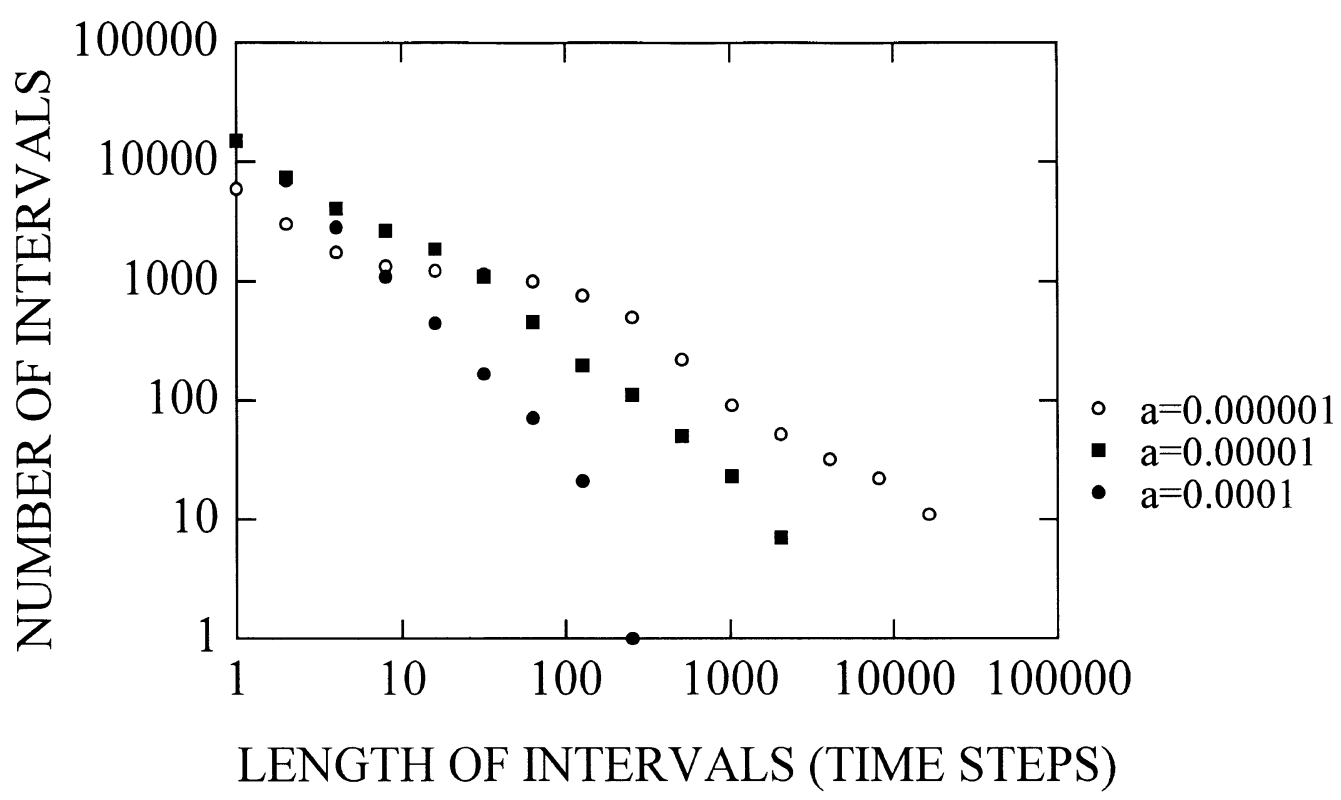

(b) $b=0.4, b o=0.0$

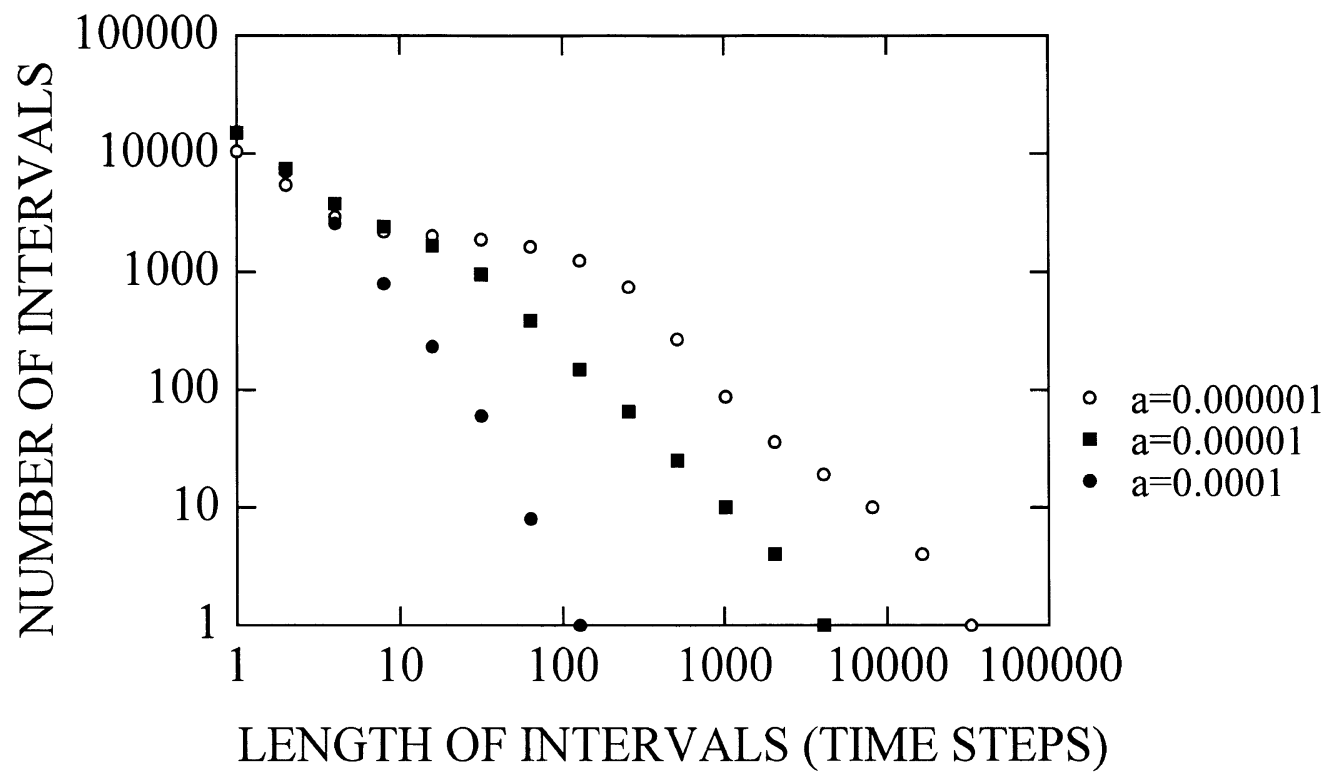

Fig. 11. The effect of parameter $a$ on the distribution of polarity reversal intervals in systems $(16 \times 16)$. (a) $b o=0.1, p=0.0$ and $b=0.4$, (b) $b o=0.0$, $p=0.0$ and $b=0.4$.

is $b$, the steeper is the slope in the distribution plot. In summary, we find that the slope of the distribution of polarity reversal intervals can be controlled by $b$ and $b o$. When we put $b o=0.05 \sim 0.1$ and $b=0.4 \sim 0.45$, we find that the value of power exponent is near -1.5 which agrees with that obtained from geomagnetic data (Figs. 9 and 10). The CML system is as sensitive to the variation of parameter $a$ as the basic map itself. When $a$ is large, the CML system behaves too chaotically. When $a$ is small, the distribution of polarity reversal intervals shows a crossover between two domains (Fig. 11). The crossover point is roughly determined by $a$, depending on the time the elementary map spends in the peri- odic domain. We thus consider the behavior observed when $a$ is too small as an artifact due to the use of piece-wise linear map for the element dynamics.

\section{Discussion}

In this paper, we improve the model of Seki and Ito (1993). The power exponent in the distribution of polarity reversal intervals found by Seki and Ito (1993) was -0.5 , in contradiction with -1.5 for the real geomagnetic data. In order to approach the value of the real exponent, we improved our model by adding more realistic features that the actual geodynamo may have. In the CML model, like in other coupled 
disk dynamo models, spins do not have discrete $(-1$ or 1$)$ but continuous values of magnitude and can reverse the polarity autonomously. The dynamics is given by a simplified discrete map obtained from the Rikitake dynamo model. Since our CML model is much less computation time consuming than other coupled disk dynamo models, we can examine the behavior for much longer time intervals, even near the critical phase-transition. As a result, we succeed in finding a set of the parameter values for which the power exponent is similar to that of the geomagnetic data. In the model, we adopted the Rikitake dynamo dynamics as local dynamics. The Rikitake dynamo reverses its polarity, but its magnetization fluctuates widely before a polarity reversal, a very different behavior from that of the dynamo. In the future, we need to improve the local dynamics of CML model. We note that short intervals are missing in the reversal data and deviate from the power-law. Some explanations are needed to explain the deviation (Marzocchi et al., 1997; McFadden and Merrill, 1993).

The Rikitake dynamo exhibits a chaotic state and reverses the polarity randomly in some parameter regions (Ito, 1980). Coupled Map Lattice was introduced by Kaneko for the study of spatiotemporal chaos (Kaneko, 1993). In our simulation, the power exponent of polarity reversal lengths is close to the experimentally found value when each element behaves chaotically. A study related to geomagnetic reversal and chaos was done also by Cortini and Barton (1994). They concluded that low-dimensional chaos can not be demonstrated in the case of the geomagnetic field. Our CML model is not low-dimensional but high-dimensional, although it is a deterministic dynamical system. The model parameter $b$ must be large while parameter $b o$ must be small, in order the model power exponent to be similar to that of the geomagnetic field reversal data. When $b$ is large, each element is in a strongly chaotic state and has large Lyapunov exponent. The evidence that the slope of power law distribution of geomagnetic reversals data is steep and is 1.5 may suggest that the dynamo in the Earth's core is turbulent, and is in a strongly chaotic state.

Acknowledgments. We would like to thank Dr. Y. Otofuji, Dr. M. Hyodo and Dr. V. B. Ryabov for valuable advice. We are very much indebted to Mr. H. Sadaoka for valuable discussions. We would like to thank Dr. M. Kono and Dr. M. Cortini for their critical comments and helpful advices to improve the manuscript.

\section{References}

Bak, P., C. Tang, and K. Wiesenfeld, Self-organized criticality: An explanation of 1/f noise, Phys. Rev. Lett., 59, 381-384, 1987.

Bak, P., How Nature Works: the Science of Self-Organized Criticality, 212 pp., Copernicus, New York, 1996.

Bergé, P., Y. Pomeau, and Ch. Vidal, Order within Chaos: towards a Deterministic Approach to Turbulence, preface by David Ruelle, translated from the French by Laurette Tuckerman, 329 pp., Wiley, New York, Hermann, Paris, 1984.

Cande, S. C. and D. V. Kent, Revised calibration of the geomagnetic polarity timescale for the Late Cretaceous and Cenozoic, J. Geophys. Res., 100, 6093-6095, 1995

Channell, J. E. T., E. Erba, M. Nakanishi, and K. Tamaki, Late Jurassic-Early Cretaceous time scales and oceanic magnetic anomaly block models, in Geochronology, Time Scales, and Global Stratigraphic Correlation, edited by W. A. Berggren, D. V. Kent, M. P. Aubry, and J. Hardenbol, pp. 51-63, Society of Sedimentary Geology Special Publication No. 54, SEPM, Tulsa, 1995.

Cortini, M. and C. C. Barton, Chaos in geomagnetic reversal records: A comparison between Earth's magnetic field data and model disk dynamo data, J. Geophys. Res., 99, 18021-18033, 1994.

Cox, A., Lengths of geomagnetic polarity intervals, J. Geophys. Res., 73, 3247-3260, 1968.

de Wijs, G. A., G. Kresse, L. Vočadlo, D. Dobson, D. Alfè, M. J. Gillan, and G. D. Price, The viscosity of liquid iron at the physical conditions of the Earth's core, Nature, 392, 805-807, 1998.

Gaffin, S., Analysis of scaling in the geomagnetic polarity reversal record, Phys. Earth Planet. Int., 57, 284-290, 1989.

Harland, W. B., A. V. Cox, P. G. Llewellyn, C. A. G. Pickton, A. G. Smith, and R. Walters, A Geologic Time Scale, pp. 73-75, Cambridge University Press, New York, 1982.

Ito, K., Chaos in the Rikitake two-disc dynamo system, Earth Planet. Sci. Lett., 51, 451-456, 1980.

Kaneko, K., The coupled map lattice: introduction, phenomenology, Lyapunov analysis, thermodynamics and applications, in Theory and Applications of Coupled Map Lattices, edited by K. Kaneko, pp. 1-49, John Wiley, New York, 1993.

Kono, M., Rikitake two-disk dynamo and paleomagnetism, Geophys. Res. Lett., 14, 21-24, 1987.

Lorenz, E. N., Deterministic nonperiodic flow, J. Atoms. Sci., 20, 130-141, 1963.

Lutz, T. M. and G. S. Watson, Effects of long-term variation on the frequency spectrum of the geomagnetic reversal record, Nature, 334, 240242, 1988.

Marzocchi, W., F. Mulargia, and G. Gonzato, Detecting low-dimensional chaos in geophysical time series, J. Geophys. Res., 102, 3195-3209, 1997.

McFadden, P. L., Statistical tools for the analysis of geomagnetic reversal sequences, J. Geophys. Res., 89, 3363-3372, 1984.

McFadden, P. L. and R. T. Merrill, Lower mantle convection and geomagnetism, J. Geophys. Res., 89, 3354-3362, 1984.

McFadden, P. L. and R. T. Merrill, Inhibition and geomagnetic field reversals, J. Geophys. Res., 98, 6189-6199, 1993.

Naidu, P. S., Statistical structure of geomagnetic field reversals, J. Geophys. Res., 76, 2649-2662, 1971.

Rikitake, T., Oscillations of a system of disk dynamos, Proc. Cambridge Philos. Soc., 54, 89-105, 1958.

Seki, M. and K. Ito, A phase-transition model for geomagnetic polarity reversals, J. Geomag. Geoelectr., 45, 79-88, 1993.

Shimizu, M. and Y. Honkura, Statistical nature of polarity reversals of the magnetic field in coupled-disk dynamo models, J. Geomag. Geoelectr., 37, 455-497, 1985.

Sornette, D., Critical phase transitions made self-organized: a dynamical system feedback mechanism for self-organized criticality, J. Phys. I France, 2, 2065-2073, 1992.

Takayasu, H., Fractals in the Physical Sciences, 170 pp., Manchester University Press, Manchester, 1990

M. Seki (e-mail: seki@kobe-tokiwa.ac.jp) and K. Ito (e-mail: ito@funhakodate.gr.jp) 